\title{
Un fundamento filosófico para un modelo de sociedad culturalmente diversa
}

\section{María del Carmen Gómez Martínez}

1 Nacionalidad: Mexicana

Grado: Maestra en Filosofía de la Ciencia

Especialización: Epistemología y diversidad cultural

Adscripción: Universidad Nacional Autónoma de México. Profesora de la Facultad de Estudios Superiores Acatlán

Correo electrónico: maricarmen.gomez.m@gmail.com

Fecha de recepción: 12 de agosto de 2011

Fecha de aceptación: 10 de agosto de 2012 


\title{
A Philosophical Basis for a Model of Culturally Diverse Society
}

\section{Resumen}

El presente artículo pretende esbozar algunas bases filosóficas que permitan fundamentar un modelo de sociedad que no deje de lado la gran riqueza que tanto étnica como culturalmente hay en nuestro país. El punto de partida es que la diversidad cultural es un hecho evidente, pero los modelos provenientes de la Modernidad han insistido en suprimir un hecho como el de la diversidad cultural en aras de una única cultura. La lucha continua de los grupos étnicos y culturales por el respeto a sus formas de vida nos lleva a pensar que tiene sentido insistir en la creación de modelos de sociedad que atiendan las necesidades de la diversidad cultural. Este modelo es sostenido por dos tesis filosóficas: la relatividad conceptual y la relatividad ontológica.

Palabras Clave: Diversidad cultural, prácticas, relatividad conceptual, relatividad ontologica.

\begin{abstract}
This article aims to outline some philosophical bases that allow support of a model of society that does not give up the ethnically and culturally richness that is in our country. The starting point is that cultural diversity is an obvious fact, but the models from the Modernity insisted on deleting an event such as cultural diversity for the sake of a single culture. The continuing struggle of ethnic and cultural groups respect their ways of life leads us to think it makes sense to insist on the creation of models of society to meet the needs of cultural diversity. This model is supported by two philosophical theses: relativity conceptual and ontological relativity.
\end{abstract}

Key Words: Cultural diversity, practice, conceptual relativity, ontological relativity. 


\section{Introducción}

$\mathrm{L}$

a gran riqueza y pluralidad étnica y cultural en México es un hecho que resulta evidente a pesar de todos los esfuerzos liberales que desde el siglo XIX se han hecho para lograr la homogeneidad de los mexicanos. Aun cuando dicha pluralidad se ha considerado como obstáculo para los propósitos liberales, no se ha logrado eliminarla, esta constituye una realidad de nuestro país. Los diversos grupos étnicos y culturales han ido adaptándose a los procesos de cambio sociales y políticos no sin cierta resistencia, pues no han dejado de exigir respeto a sus formas de vida, no han dejado de exigir "el derecho a la igualdad cuando la diferencia los inferioriza y a la diferencia cuando la igualdad los descaracteriza" (Sousa, 1997:52).

La lucha de estos grupos étnicos y culturales puede fundamentarse en un modelo de sociedad que se apegue a las condiciones reales en que viven estas culturas en México y que integre las necesidades y retos de una sociedad culturalmente diversa como, por ejemplo, la posibilidad de una auténtica interculturalidad. De esta manera, la reflexión que presentamos está encaminada a identificar ciertas bases filosóficas sobre las que podría apoyarse dicho modelo, a saber: la tesis de la diversidad cognoscitiva. En otras palabras, estos grupos exigen que se respete su forma de vida y su lucha puede sustentarse en la tesis de que no existe un único sistema de conocimientos o valores para todos; que las personas tienen razones para vivir y concebir el mundo en el que viven de modos diferentes.

La estructura que sigue este texto es la siguiente: señalaremos que la diversidad cultural se hace evidente en las prácticas sociales de los diferentes grupos étnicos y culturales. A continuación señalaremos que uno de los elementos de dichas prácticas son los esquemas conceptuales y, más adelante, mostraremos que la coexistencia de esquemas conceptuales diferentes se justifica por medio de una postura relativa tanto en el ámbito conceptual como en el ontológico. Finalmente concluiremos, si la argumentación presentada lo permite, que no existe un sistema de conocimientos único y de validez universal, sino que existen conceptualizaciones del mundo que marcan cosmovisiones parti- 
culares y que la forma como se concibe el mundo está directamente relacionada con la forma como viven los sujetos en el mundo.

\section{I}

La diversidad cultural asume que diferentes grupos humanos no pueden concebirse como culturalmente homogéneos; existen diferencias que están constreñidas por una cultura en particular, es decir, están sujetas a formas específicas de vida alrededor de las cuales se constituye un grupo de personas. La conformación de un grupo de personas se lleva a cabo en las prácticas sociales; en ellas, los sujetos se relacionan e interaccionan al mismo tiempo que van creando y recreando su cultura. En los términos de Parekh:

las creencias que sostienen los seres humanos sobre el sentido y el significado de la vida humana, así como sobre las actividades y relaciones que forman parte de ella, configuran las prácticas en torno a las cuales estructuran y regulan sus vidas individual y colectivamente. Utilizaré el término cultura para referirme a estos sistemas de creencias y prácticas. $(2006: 142)$

Si la cultura puede considerarse como un sistema de prácticas y creencias, entonces la pluralidad cultural se manifiesta a través de las diferentes creencias que sostienen y las diferentes prácticas que llevan a cabo los sujetos que pertenecen a diferentes culturas. Sin embargo, cabe señalar que la diversidad cultural no es un hecho simplista en el que los sujetos tienen una variedad de opiniones o aspiraciones, si no que implica diferencias significativas en cuanto al sistema de creencias y prácticas sociales que conforman las culturas. Aunque existen diferentes formas de diversidad cultural y una gran cantidad de países declarados multiculturales, en este trabajo consideraremos aquella forma que nos remite a culturas encarnadas en pueblos indígenas cuyos orígenes fueron pueblos prehispánicos.

En este sentido, vale la pena dar cuenta de cómo están constituidas las prácticas; para ello seguiremos la idea de Theodore Schatzki. Según este autor, podemos entender las prácticas como "un conjunto de 
hechos y dichos, organizado por un grupo de acuerdos, un conjunto de normas y una estructura teleoafectiva" (2001:53). De esta forma, los sujetos que participan de una práctica llevan acciones como consecuencia del conjunto de reglas, valores, fines, creencias, proyectos, objetivos e incluso emociones que les constituyen.

De acuerdo con esta concepción tres son los elementos que se destacan en la constitución de una práctica. El primero de ellos tiene que ver con las habilidades pertinentes para determinadas acciones en las circunstancias ofrecidas por el medio, es decir, se trata de habilidades que se expresan en un "saber cómo": saber cómo hacer algo, saber cómo identificar algo, saber cómo responder ante algo, saber cómo demostrar algo, saber cómo enunciar algo, etc. Esto es, el tipo de acciones que constituyen una práctica se ven reflejadas en acciones como 'hacer', 'identificar', 'responder', 'demostrar', 'enunciar', etc. Por ejemplo, las acciones que constituyen una práctica lingüística están expresadas por la capacidad de usar las palabras, seguir reglas, identificar funciones, elaborar juicios, llevar a cabo inferencias y cosas parecidas. Lo cual, en última instancia, nos remite al conjunto de sujetos que - se presupone - tienen capacidades y/o habilidades específicas para llevar a cabo dichas acciones.

El segundo elemento, el de las reglas, se refiere a formulaciones explícitas que permiten y prohíben acciones particulares. Schatzki señala que lo que la gente normalmente hace refleja su propio entendimiento de reglas específicas. Es necesario agregar que, dado que las prácticas responden a contextos específicos, la normatividad - los conjuntos de reglas, instrucciones y direcciones - se va constituyendo al interior de las prácticas; por ejemplo, en la práctica lingüística el agente da cuenta de que sabe usar correctamente las palabras cuando estas son adecuadas al contexto. $Y$, en este sentido, nuestros términos evaluativos, i.e., aquellos que señalan lo que es correcto o aceptable tendrían que definirse en función de los fines y valores, de ahí que el tercer elemento debamos entenderlo como una normatividad valorativa.

El tercer elemento que el autor señala como una mezcla de teleología y afectividad está enfocado hacia una cuestión de fines y valores. Esto es, una persona lleva a cabo acciones de manera intencionada y 
en un momento específico de acuerdo a las creencias que posee, pero el tipo de acciones que puede llevar a cabo depende en gran medida de los fines que persiga y de cómo tendría que proceder para alcanzar dichos fines, lo cual está muy ligado con sus creencias, deseos y expectativas. Esto es algo que Schatzki llama 'inteligibilidad práctica' y que, de alguna manera, determina la forma de la actividad humana pues una persona lleva a cabo las acciones que para ella tienen sentido o son significativas, pero tienen sentido en la medida en que están determinadas por la estructura teleo-afectiva. En el caso de la práctica lingüística estaríamos considerando el uso que una comunidad hace de los términos, de la gramática y las conductas asociadas a estas con propósitos específicos, i.e., conductas que reflejan que sí hay un seguimiento de las reglas establecidas por la comunidad.

Estos tres elementos si bien no determinan las acciones que se llevan a cabo al interior de una práctica - pues es posible que existan otros factores que incidan en las acciones de los sujetos- al menos dan cuenta en términos estructurales de la constitución de estas ( $c f$. Schatzki, 2001:47-53). Esto se debe a que las prácticas están sujetas a intereses, valores, tradiciones, lenguajes o historias específicas, i.e., varían de acuerdo con el tiempo y las circunstancias. Esta variabilidad da cuenta de que las prácticas no están determinadas y de que son los mismos individuos los que las van creando y recreando, y en esa medida es que los sujetos se van constituyendo a sí mismos.

De acuerdo con esta idea schatzkiana de práctica existe un conjunto de elementos que constriñen o posibilitan la acción de los sujetos, mismo que puede considerarse como el esquema conceptual de dichos sujetos. El que los sujetos tengan determinadas creencias, valores, intereses, fines o reglas obedece a un esquema conceptual determinado. De modo que es suficiente con llevar a cabo un análisis de las prácticas que realizan los sujetos que pertenecen a una cultura para mostrar sus esquemas conceptuales. Dicho de otro modo, analizar las prácticas no solo nos revelaría ese aspecto normativo y teleleo-afectivo, sino que además daría cuenta del modo en que la gente es en las prácticas. 
En el apartado anterior señalábamos la idea de que la manera de concebir el mundo está condicionada por los esquemas conceptuales, esto quiere decir que la manera como organizamos conceptualmente la realidad tiene como supuesto fundamental los esquemas conceptuales; desde estos es que se puede llevar a cabo dicha organización y también desde estos puede establecerse la normatividad que constriñe las acciones de los individuos que comparten un esquema conceptual. No obstante, habría que señalar desde ahora que el propósito de hablar de marcos conceptuales es meramente analítico, la idea de marco conceptual es solo una parte constituyente de las prácticas. Es, por decirlo de alguna manera, la parte teórica-normativa que nos permite articular la acción. Por ello, vale la pena dar cuenta de lo que entendemos por marco o esquema conceptual.

La noción de marco conceptual es análoga, en cierto sentido, a la noción de paradigma en el sentido kuhniano. Kuhn cuando habla de la ciencia normal señala que esta es una etapa en la que los científicos trabajan bajo un marco de supuestos que circunscribe cuáles son los elementos de la naturaleza a los cuales se remite, cuáles son los problemas importantes, cuáles son los métodos de justificación y validez del conocimiento, cuáles son los criterios de evaluación, etc. (cf. Kuhn, 1971:84). Siguiendo esta idea, las nociones de paradigma como la de esquema conceptual cumplen una función muy importante: delimitan un mundo con sus propios problemas, métodos o criterios de evaluación, valores, fines y ontología. Desde esta perspectiva, el esquema conceptual puede ser pensado como una estructura que constriñe o condiciona el conocimiento mismo.

Si esto es cierto y si aceptamos que las prácticas no están determinadas, sino que se van constituyendo a través de la continua interacción de los sujetos que forman parte de ellas, entonces todos los elementos constituyentes (sean estos epistémicos, metodológicos, axiológicos, lógicos, normativos, etc.) dependen o se definen al interior de las mismas prácticas. Esta dependencia nos conduce a la creencia de que los contenidos de estos elementos no son universales y necesarios de todo marco conceptual, sino contingentes. En otros términos, estos 
elementos no necesariamente son comunes a todos los esquemas conceptuales y aunque estuvieran presentes en la mayoría de ellos habría que señalar que los contenidos pueden variar: la noción de lo que es la racionalidad, por poner un ejemplo, puede ser muy diferente en dos esquemas conceptuales.

Asumir que nuestros conceptos son relativos a los esquemas conceptuales ha generado una serie de dudas respecto de qué tanto podemos o no evaluar de 'racionales' (o en su caso de irracionales) las conductas de sujetos que pertenecen a otras culturas y que tienen diferentes esquemas conceptuales. En este sentido resulta interesante la propuesta que hace Winch (1994) respecto de la relatividad de nuestros estándares de inteligibilidad, al señalar que el origen de dichos estándares está en las instituciones sociales mismas.

Winch argumenta que si cuando evaluamos algo de 'racional' esta evaluación la hacemos desde nuestra comprensión de los que es o no racional, entonces resulta absurdo decir que algo nos resulta racional o no cuando los estándares de racionalidad de las diferentes culturas no coinciden. La idea subyacente es que al evaluar a los sujetos que no comparten nuestros 'estándares de inteligibilidad' solo puede resultar fructífera en la medida en que no nos empeñemos en "invocar nuestras propias normas de racionalidad" y estemos dispuestos a ampliar nuestro modo de ver el mundo. De ahí que Winch afirme que "estudiar seriamente otro modo de vida es necesariamente buscar la ampliación del nuestro"(Winch, 1994: 65).

III

De acuerdo con los dos apartados anteriores los esquemas conceptuales nos permiten constituir el mundo de una u otra forma, lo cual no significa que tengamos "diferentes alternativas conceptuales para un único Mundo" (Gómez, 2009: 23), significa que, en virtud de los marcos o esquemas conceptuales, hablamos de mundos reales, ontológica y conceptualmente diferentes. Sin embargo, esta idea puede llevarnos a suponer que es imposible la diversidad cognoscitiva, es decir, que es imposible comprender, justificar o incluso evaluar los procesos de conocimiento que lleva a cabo un sujeto que no comparte nuestro 
esquema conceptual. Esta idea proviene del supuesto de que si dos esquemas conceptuales son en realidad diferentes, entonces son inconmensurables, en el sentido de que no son comparables objetivamente y por ende, tampoco pueden ser evaluados. Pero esta es una postura radical que conduciría a señalar una suerte de relativismo con respecto al propio esquema conceptual y que, en esa medida, "todo vale" pues está justificado de acuerdo con los criterios que establece un esquema conceptual. Un relativismo extremo como este resulta inconsistente.

En el caso que nos ocupa el propósito es mucho más limitado, se trata de justificar un modelo de sociedad en el que coexistan diversos sistemas de conocimiento a través de una postura relativa, mas no relativista. $\mathrm{Al}$ asumir una forma de relatividad se justifica la idea de que existen diferentes maneras de concebir el mundo y con ello, diferentes maneras de vivir en él. Pero si vamos más lejos, podríamos asumir que estas diferencias no anulan la interacción entre sistemas de conocimiento que subyacen a diferentes culturas.

Así, en lo que sigue desarrollaremos la idea de la diversidad cognoscitiva apoyándonos en dos tesis epistemológicas que son constitutivas de aquella: la relatividad ontológica y la relatividad conceptual. Es necesario señalar que se hace la distinción entre el nivel epistemológico y el nivel ontológico con fines analíticos, pero no debe perderse de vista que ambos niveles se encuentran relacionados de una manera muy cercana, pues es desde el ámbito del discurso que podemos referirnos a la realidad y esto es algo que se constata en las mismas prácticas.

La tesis de la relatividad conceptual asume que no existe un sistema conceptual que sea el mejor o el más adecuado para organizar conceptualmente la realidad. La pretensión de que existe un marco conceptual que nos permite describir de forma verdadera la realidad se ha considerado errónea. Putnam ejemplifica muy claramente este tipo de propuesta cuando señala que nuestros conceptos no tienen un significado absoluto y por consiguiente, no podemos afirmar que existe una sola interpretación de estos. Putnam se refiere a la relatividad conceptual en los siguientes términos: "los mismos conceptos lógicos primitivos, y en particular las nociones de objeto y existencia, tienen 
una multitud de diferentes usos más que un «significado» absoluto" (Putnam, 1994:64).

$\mathrm{Si}$, de acuerdo con estos señalamientos, identificar un objeto como objeto depende de nuestro esquema conceptual, así como el significado y uso dependen también de este, entonces cualquier término debería siempre definirse al interior del esquema conceptual. Por ejemplo, veamos lo que sucede con la noción de 'verdad'. Si aceptamos la postura putnamiana, nos vemos imposibilitados a hablar de la 'verdad' en el mismo sentido en que lo plantea una postura externalista, a saber: como una correspondencia única y natural entre los objetos del mundo y las categorías o conceptos con que nos referimos a ellos. Al aceptar el planteamiento de Putnam, tendríamos que rechazar la versión del externalista y entonces, bien podría uno cuestionarse qué tipo de relación hay entre lo que decimos del mundo (la teoría) y el mundo mismo (la realidad).

Desde una perspectiva internalista (que asume cierta relatividad) y realista, como la que suscribimos, la respuesta a esta interrogante sería que todo objeto está conceptualmente constituido. No obstante, no es una mera invención de la mente humana pues no podemos prescindir de los insumos que nos provee la experiencia. De ahí que Putnam afirme que "los objetos no existen independientemente de los esquemas conceptuales" (1981:52).

El trasfondo es evidentemente kantiano: Putnam retoma de Kant la idea de que los 'objetos externos' (cosas-en-sí) que percibimos son una representación fenoménica (cosas-para-nosotros) que resulta de sintetizar las sensaciones (elemento empírico) y la Sensibilidad (facultad del sujeto). Según Kant, la sensibilidad es la capacidad de recibir representaciones, de modo que todo 'objeto' para que pueda ser percibido está sujeto a las condiciones de la Sensibilidad (que son las formas a priori del espacio-tiempo). Por eso es que no percibimos simplemente "objetos-en-sí" (que no están constituidos dentro de algún esquema conceptual), sino "objetos-para-nosotros" (Kant, 1978: A1950/B33-74).

Cuando un internalista dice algo respecto del mundo efectivamente asume un compromiso ontológico, pero la forma en que se refiere 
al mundo depende del esquema conceptual. Por eso la "verdad" para el internalista no es cuestión de correspondencia entre nuestros juicios y lo que existe 'como tal' en el mundo, sino que se trata de una justificación: es "un tipo de coherencia de y entre nuestras creencias y con nuestras experiencias tal como estas se nos presentan en nuestro sistema de creencias" (Putnam, 1981: 50).

Esto, que el autor señala como 'aceptabilidad racional idealizada', tiene dos acotaciones: por un lado, la aceptabilidad racional es contextual, lo cual quiere decir que es relativa a una persona y a un tiempo, y por ello es necesaria mas no suficiente, pues de lo contrario podría decirse que se trata de un relativismo que tiende a ser extremo. Por otro lado, la aceptabilidad racional debe ser idealizada, lo cual quiere decir que se exigen ciertas condiciones epistémicamente ideales. Desde este supuesto, la verdad depende de ciertas condiciones ideales, pero ¿qué debe entenderse por condiciones ideales? Si lo ideal se piensa como aquello que es inalcanzable, entonces nos comprometemos con una justificación que va más allá de los mismos esquemas conceptuales ( $c f$. Pérez, 1992:89); pero, en la medida en que lo ideal se piensa como lo óptimo, como una situación óptima de afirmación, entonces el compromiso no es tan fuerte. En una versión en la que Putnam toma como referencia lo óptimo señala que:

Si yo digo 'hay una silla en mi estudio', una situación epistémica ideal sería la de estar en mi estudio con las luces encendidas o con la luz del día entrando a través de la ventana, sin ningún problema con mi vista, con una mente que no esté confundida, sin haber ingerido drogas o haber estado sujeto a hipnosis, etcétera, y mirar a ver si ahí hay una silla. (Putnam, 1990: viii)

La idea subyacente a este criterio de lo que es la verdad es que si bien tenemos razones para aceptar una proposición no es suficiente con la aceptación, sino que esta debe tener una base que la constriña: la realidad misma. Por ello señala Putnam que aunque podamos aceptar como razonable la proposición 'hay una silla en mi estudio', habría que 'mirar a ver si ahí hay una silla'. 
Si no tuviéramos el constreñimiento de la realidad, podríamos crear historias o meras construcciones lingüísticas que no den cuenta de los fenómenos de la realidad que está ahí y que no es construida por nosotros. Así, cuando una teoría es verdadera no es simplemente porque pueda o no estar justificada, sino porque describe de algún modo la realidad. En este punto no hay que perder de vista que, históricamente, la noción de verdad como adecuación con la realidad ha encaminado la investigación: "la elucidación de las pretensiones de verdad y el reconocimiento del peso que estas tienen en la investigación científica, nos revelan el papel fundamental que cumple la verdad como idea regulativa" (Pérez, 1993: 60).

En suma, si los insumos de la experiencia están formados en cierto grado por nuestros conceptos, entonces cuando aseveramos algo lo que hacemos es señalar el hecho como tal —existente independientemente de que sea enunciado, que es lo que hace que esta postura sea realista-, pero ese hecho es enunciado de acuerdo a como nuestro esquema conceptual recorta los hechos en el mundo - que es lo que hace de esta un postura relativa e interna.

Hasta ahora hemos considerado la tesis de la relatividad conceptual en estos términos: nuestras nociones o conceptos no tienen significados absolutos, sino que son relativos a esquemas conceptuales. Pero existe otro elemento que acompaña a este tipo de relatividad y es la tesis de 'inconmensurabilidad'. Si nos tomamos en serio esta tesis, entonces podríamos decir que en dos esquemas conceptuales (EC1 y $\mathrm{EC} 2$ ) existen creencias básicas y diferentes de tal manera que tienen alcance y consecuencias empíricas diferentes. Si estas diferencias son significativas entonces EC1 y EC2 son inconmensurables, es decir, no podríamos definir algunas de las creencias de $\mathrm{EC} 1$ en términos de EC2.

Si hacemos una analogía con la dinámica científica que nos plantea Kuhn, este tipo de inconmensurabilidad es como la que ocurre entre dos paradigmas científicos que incluyen modelos de problemas y soluciones que son aceptados por una comunidad científica en una época determinada. $\mathrm{Y}$ de esto podemos dar cuenta a través de los ejemplos que nos provee la historia de la ciencia. Según Kuhn, en la historia de 
la ciencia podemos encontrar cambios revolucionarios a los que subyacen cambios tanto en el nivel conceptual como en el nivel ontológico. Por ejemplo, en el caso de la transición de la astronomía ptolemaica a la copernicana dice que:

Antes de que esta transición tuviera lugar, el Sol y la Luna eran planetas, pero la Tierra no. Después la tierra era un planeta como Marte y Júpiter; el Sol era una estrella; y la luna era un tipo nuevo de cuerpo, un satélite. Cambios de esta clase no fueron correcciones de errores individuales englobados en el sistema ptolemaico [...] esos cambios incluían no sólo cambios en las leyes de la naturaleza, sino también cambios en los criterios mediante los que algunos términos de esas leyes se conectaban con la naturaleza. Cuando este tipo de cambios de referentes acompaña un cambio de ley o de teoría, el desarrollo científico no es completamente acumulativo. No se puede pasar de lo viejo a lo nuevo mediante una simple adición a lo que ya era conocido. Ni tampoco se puede describir completamente lo nuevo en el vocabulario de lo viejo o viceversa. (Kuhn, 1989: 59-60)

Este tipo de ejemplos muestran dos cosas: por un lado, que las diferencias que existen en los procesos de cambio científico muestran que no hay una teoría que describa verdaderamente la realidad y, por consiguiente, si hacemos una revisión histórica podemos encontrar diversas concepciones. Esto se debe a que hay una estructura léxica de la cual depende la descripción del mundo. Para Kuhn, esa estructura o taxonomía es una condición previa, no solo para la descripción del mundo sino incluso como condición de comunicabilidad al interior de una comunidad y como identificación respecto de otras comunidades.

Sin embargo, esto no implica que sean 'lógica y psicológicamente anteriores a los objetos y situaciones para los que sirven como estructura'; tanto estructuras como objetos se aprenden a la vez. Es así que: "la presencia de masas y fuerzas es un criterio para lo que podría llamar una "situación mecánico-newtoniana", es decir, una en la que se aplica la segunda ley de Newton. Pero puede aprenderse a reconocer masa y fuerza solo dentro de la situación mecánico-newtoniana y viceversa" 
(Kuhn, 1989: 129). Esta manera de concebir el 'mundo real' presupone a un mismo tiempo el objeto como la estructura. De tal manera que Kuhn bien podría afirmar - junto con Putnam - que los objetos son tanto hechos como descubiertos.

$\mathrm{Si}$ es cierto que el mundo es experimentado a través de diferentes léxicos, entonces la diferencia de experiencias podría mostrar una invención o creación por parte de quienes lo habitan, pero al respecto Kuhn declara que:

... la metáfora de la invención, construcción y subjetividad son groseramente engañosas en dos sentidos. Primero, el mundo no es inventado, ni construido. Las criaturas a las que se imputa esta responsabilidad ya encuentran el mundo ahí $[. .$.$] este mundo ha sido experiencialmente dado,$ en parte directamente a los nuevos habitantes y, en parte indirectamente por herencia. (Kuhn, 2002: 126)

Ciertamente, el mundo está ahí, pero la condición de posibilidad de una experiencia parece estar dada por el léxico. De esta manera, Kuhn establece una analogía con las categorías kantianas pues son estas condición de posibilidad de toda experiencia, solo que para Kant esas categorías son universales. Y Kuhn presupone que "las categorías léxicas, a diferencia de las kantianas, pueden cambiar y lo hacen tanto con el tiempo como de una comunidad a otra" (Kuhn, 2002: 129).

Por otro lado, estos ejemplos señalan que las diferencias que se derivan de estas concepciones alcanzan un nivel ontológico en la medida en que nos remiten a entidades diferentes o incluso inexistentes cuando se cambia de paradigma. Y por ello es que surge la inconmensurabilidad; esta se presenta en la medida en que no se puede llevar a cabo una descripción adecuada de los referentes de un término que pertenece a otro esquema conceptual, pues justamente la labor de comprensión consiste en trazar hipótesis que den sentido y coherencia a los pasajes que resultan ininteligibles y eso requiere, en el menor de los casos, reconocer y describir características de los referentes siempre 
y cuando sea posible (Kuhn, 1989: 106). ${ }^{1}$ Cuando no es posible, entonces se busca aprender nuevos términos, pero este proceso de aprendizaje no es de uno en uno, ya que muchos de ellos son términos que pertenecen a conjuntos interrelacionados o son términos que solo pueden aprenderse junto con la teoría a la que pertenecen. No obstante, es necesario señalar que el problema de la inconmensurabilidad no se resuelve añadiendo un nuevo término al lenguaje, sino que es necesario re-estructurar la taxonomía con la que se cuenta.

Este caso que señala Kuhn anuncia ya la tesis de la relatividad ontológica, la cual —insistimos - va muy de la mano con la tesis de la relatividad conceptual, y dice que los objetos que existen en el mundo dependen ontológicamente de la conceptualización que lleva a cabo la mente. En este sentido Putnam afirma que

Los «objetos» no existen independientemente de los esquemas conceptuales. Recortamos el mundo en objetos cuando introducimos uno u otro esquema descriptivo, y puesto que tanto los objetos como los signos son internos al esquema descriptivo, es posible indicar cómo encajan. (Putnam, 1981: 52)

Como puede apreciarse, este tipo de relatividad ontológica también está sustentada en una postura internalista puesto que nuestros marcos o esquemas conceptuales llevan consigo un compromiso ontológico con determinados objetos, i.e., son ellos - los esquemas con-

1 En el mismo tenor, Winch señala que en la medida en que queramos comprender 'otro mundo', 'otro esquema conceptual', tendríamos que ser nosotros mismos quienes ampliemos nuestra comprensión de modo que podamos darle cabida a esa otra forma de vida. En la crítica que hace a MacIntyre respecto de cómo hacernos inteligible la vida de los zande, Winch afirma lo siguiente: "las formas de vida de los zande no deben evaluarse y clasificarse del modo que MacIntyre afirma: a partir de ciertas formas de vida específicas que se encuentran en nuestra cultura, es decir, según que las formas de vida de los zande puedan medirse o no con las exigencias de las formas de vida de nuestra cultura [...] Dado que somos nosotros los que queremos comprender la categoría zande, nuestra es la responsabilidad de ampliar nuestra comprensión de modo que demos cabida en ella a la categoría zande, más que insistir en verla en términos de nuestra propia distinción elaborada entre ciencia y no-ciencia”(Winch, 1994: 70-1). 
ceptuales - los que delimitan qué entidades, procesos y/o principios son aceptables. Por eso a la pregunta de '¡cuáles son los objetos que constituyen el mundo?' Putnam responde que eso depende del esquema conceptual que uno tenga. Si esto es así, entonces hay que asumir que no hay objeto fuera de un marco conceptual, pero también que es posible que exista un objeto del cual un marco conceptual no puede dar cuenta y otro sí.

Ahora bien, aunque Putnam admita una dependencia de los esquemas conceptuales en un sentido plural, pues no hay tal cosa como 'el esquema conceptual', no cae en un tipo de constructivismo extremo en el que la realidad independiente no contribuye de ningún modo a la formación del esquema conceptual. Esto es algo que ya habíamos señalado, pero que vale la pena enfatizar, pues el autor señala que "los objetos mismos son tanto hechos como descubiertos, tanto productos de nuestra invención conceptual como del factor 'objetivo' en la experiencia" (Putnam, 1981: 54). Con lo cual no se trata de rescatar un objeto ya dado, en sí-mismo, pero tampoco se trata de un constructivismo extremo que no se sustenta en la realidad ni está constreñida por ella.

En suma, los supuestos básicos en los que se sostiene la relatividad ontológica y epistémica serían: (1) la identificación de un objeto como tal depende de nuestro esquema conceptual y por ende, (2) todas las teorías que se derivan de nuestra forma de concebir el mundo son verdaderas, en parte, porque describen la realidad, y finalmente, (3) la idea de verdad en la que se apoya (2) tiene un trasfondo kantiano en el sentido de que la 'verdad' no puede ser considerada como correspondencia con una realidad pre-estructurada. Kant asume la existencia de una realidad independiente como un postulado de la razón, y se refiere a ella como las cosas en sí-mismas o noúmena, pero los noúmena no son más que el límite de nuestro pensamiento del cual no podemos formarnos concepción alguna. Al mismo tiempo hablar de objetos empíricos, que no es hablar de objetos en sí-mismos, sino de fenómenos, significa hablar de objetos para nosotros.

En la "Estética Trascendental", Kant señala lo siguiente: como fenómenos no pueden existir en sí mismos, sino solo en nosotros. Permanece para nosotros absolutamente desconocido que sean los objetos 
en sí, independientemente de toda esa receptividad de nuestra sensibilidad”.

Y más adelante señala que:

sostener que toda nuestra sensibilidad no es más que la confusa representación de las cosas, una representación que sólo contendría lo que pertenece a las cosas en sí mismas, pero que las contendría en una masa de características y representaciones parciales que no distinguimos conscientemente, constituye una falsificación de los conceptos de sensibilidad y fenómeno, una falsificación que inutiliza y vacía toda la teoría relativa a estos conceptos. (Kant, 1978, A42-A44 o B59-B61)

Estas dos citas muestran claramente el compromiso de todo internalista: el compromiso con una noción de fenómeno (objeto empírico), pero no entendido como representación parcial del objeto-en-símismo, sino como algo que es posible gracias a nuestra sensibilidad, como facultad inherente al sujeto. Si no lo entendemos en estos términos, dice Kant, no hemos entendido los conceptos de fenómeno, sensibilidad y experiencia, pues pretender que detrás del fenómeno hay un objeto-en-sí que es el que realmente existe, sería tanto como pretender que tenemos la experiencia de Dios. En otras palabras, si detrás del fenómeno no hay ese objeto en sí mismo, entonces bien podríamos preguntarnos para qué hace Kant la distinción entre fenómenos y noúmenos y quizá la respuesta más simple a esta interrogante es que se trata de una distinción que pretende mostrar las capacidades cognitivas del sujeto frente a la capacidad divina que (por principios teológicos) es omnisapiente, omnipotente y omnipresente, por lo que la pretensión de que podemos alcanzar el noúmeno sería como adoptar, en términos de Putnam, el punto de vista del Ojo de Dios.

\section{Conclusión}

Para terminar, retomemos algunos puntos importantes del cuerpo de este trabajo que no solo nos permitirán recapitular y concluir, sino en- 
trelazar los tres apartados. Primero, Kuhn hace un acotamiento significativo respecto de la inconmensurabilidad y dice que se halla a nivel de las estructuras taxonómicas, señalando con ello que no podemos hacer una comparación punto por punto entre las proposiciones que se expresan en el léxico de una comunidad y las proposiciones que se expresan en el léxico de otra comunidad. Es decir, no hay "traducibilidad" entre las proposiciones de diferentes mundos porque no describen los mismos objetos. Y no describen los mismos objetos justamente porque estos dependen en gran medida de la estructura o esquema conceptual. Pero no cancela, en modo alguno, la posibilidad de comunicación entre comunidades que conciben mundos "distintos" en virtud de sus esquemas conceptuales.

Con esto no quiere decirse que construimos el mundo solo y exclusivamente a partir de nuestros esquemas conceptuales, pues eso implicaría que las estructuras que podemos encontrar en la realidad no contribuyen a esa constitución del mundo. Pero Kuhn asume, al igual que Putnam, que efectivamente hay un mundo "experiencialmente dado"y que constriñe o restringe lo que podemos decir de él. Así, los objetos que hay en el mundo dependen de la estructura conceptual, pero no solo de esta. De este modo, si la constitución del mundo depende de las estructuras conceptuales habría que apuntar, para no caer en un relativismo extremo, que la realidad misma puede concebirse como la base que permite el acceso a otras estructuras conceptuales.

Segundo, la propuesta de Putnam de que los objetos son constituidos conceptualmente y de que, en esa medida, no existe una única, verdadera y completa descripción de cómo es el mundo, sino que, en todo caso, existe más de una descripción del mundo que permite afirmar que existen diferentes maneras de concebir el mundo. Sin esta suerte de relatividad ontológica y conceptual que propone Putnam, sería difícil aceptar la tesis de que existen múltiples formas de concebir el mundo.

De esta manera, si bien es cierto que la postura de Putnam nos ha permitido reconstruir la tesis de la diversidad cognoscitiva en términos de la coexistencia de dos esquemas conceptuales que no son radicalmente inconmensurables, es a través de los casos que propone Kuhn 
que podemos ir del plano teórico al práctico. Por ello es que si nuestras descripciones de cómo es el mundo resultan en formas de vida diferente es porque responden a intereses y propósitos específicos, lo cual - en última instancia - viene a enfatizar que no existe una forma privilegiada tanto para acceder al mundo como para dar cuenta de él.

Tercero y último, en el primer apartado de este escrito señalamos que las acciones que llevamos a cabo los sujetos están cargadas de intenciones, de creencias, de fines, de valores, de normas, etc., y en la medida en que atienden a diferentes valores o propósitos dan lugar a diversas formas de vida. De tal manera que las acciones no son más que un reflejo de la estructura que constituye a la práctica como tal. En diferentes países contamos con diferentes culturas tradicionales indígenas que bien podrían ejemplificar el hecho de que comunidades con esquemas conceptuales específicos les permiten concebir el mundo de una manera determinada y eso se muestra a través de sus diferentes prácticas: epistemológicas, sociales, políticas, lingüísticas, laborales, rituales, entre otras. Este ejemplo también sirve como punto de referencia al afirmar que la tesis de la diversidad cognoscitiva, que aquí hemos tratado de sustentar a través de dos tesis como lo son la relatividad ontológica y epistemológica, no es una propuesta que se queda en el nivel teórico.

Si la defensa de la tesis de la diversidad cognoscitiva es acertada, i.e., si las personas tienen razones para concebir el mundo en el que viven de modos diferentes, en función de sus distintos sistemas de creencias, entonces parece que hay razones para defender sus formas de vida. Lo cual no significa en modo alguno que se cancele la posibilidad de una autentica interculturalidad. La idea de la diversidad cognoscitiva se salva de caer en una suerte de relativismo extremo en el cual la comunicación entre culturas se antoja imposible.

\section{Referencias bibliográficas}

Bonfil, Guillermo. México Profundo. México: Random House Mondadori, 2006, p. 250. 
Concheiro, Luciano y Francisco López. (coords.) Biodiversidad y conocimiento tradicional en la sociedad rural. México: CEDRSSACámara de diputados-LIX legislatura. 2006, pp. 39-78.

Hale, Charles. El liberalismo mexicano de la época de Mora. México: S XXI, 2005, p. 347.

Kant, Immanuel. Crítica de la razón pura. Madrid: Alfaguara, 1978, p. 692.

Kuhn, Thomas. La estructura de las revoluciones cientificas. México: F.C.E., 1971, p. 319.

- ¿¿Qué son las revoluciones científicas? y otros ensayos. Barcelona: Paidós I.C.E/U.A.B., 1989, p. 161.

-. "El camino desde la Estructura", en: Conant y Haugheland (comps.). Thomas Kubn. El camino desde la Estructura. Ensayos Filosóficos 1970-1973. Barcelona: Paidós Básica, 2002, pp. 113129.

Parekh, Bhikhu. Rethinking multiculturalism. Cultural Diversity and Political Theory. New York: Palgrave Macmillan, 2006, p. 409.

Pérez, Ana Rosa. "Verdad y justificación", en: Dianoia, número 38. 1992.

—, "El realismo de Villoro", en: E. Garzón y F. Salmerón (eds.), Epistemología y Cultura. En torno a la obra de Luis Villoro, México: UNAM, 1993, pp. 39-64.

Putnam, Hilary. Reason, Truth and History. Cambridge University Press, 1981, p. 222.

-. Realism with a Human Face. Harvard University Press, 1990.

- . Las mil caras del realismo. Barcelona: Paidós, 1994, pp. 151.

Schatzki, Theodore. "Practice Mind-ed Orders", en: Theodore Schatzki, Karin Knorr Cetina y Eike von Savigny (eds.). The Practice Turn in Contemporary Theory. Nueva York: Routledge, 2001.

Sousa, B. "Una concepción multicultural de los derechos humanos", en: Memoria, No. 101, México, 1997, pp. 41-53.

Winch, Peter. Comprender una sociedad primitiva. Barcelona: Paidós I.C.E./U.A.B., 1994, p. 167. 